\title{
Line Space Theory of Resonant Four-Wave Mixing: New Prospects for All-Optical Studies of Photofragment States
}

\author{
A.P. Kouzov ${ }^{\mathrm{a}, *}$, P.P. Radi ${ }^{\mathrm{b}}$ \\ ${ }^{a}$ Department of Physics, Saint-Petersburg State University, Ulyanovskaya str. 3, \\ Peterhof, Saint-Petersburg 198504, Russia \\ ${ }^{b}$ Paul Scherrer Institute, 5232 Villigen, Switzerland
}

\begin{abstract}
Based on the line-space quantum formalism, the potential of Resonant FourWave Mixing spectroscopy as a new tool to study rotational and translational anisotropy of photofragments produced by absorption of plane-polarized photons is theoretically addressed. Synergy of the flexible polarization setup, fine quantum state resolution and of the possibility to study translational recoil distributions, makes the tool unsurpassed among the all-optical means to interrogate the photofragment states. It allows to directly separate signals induced by the rotational anisotropy which remain silent in the most of laserinduced fluorescence responses and thus opens new ways to study rotational helicity, a crucial signature of the photolysis pathway.
\end{abstract}

Introduction. - Reaction microdynamics, "the heart of chemistry" [1] manifests in the properties of nascent particles, which offers thereby considerable scope to track the reaction pathway. Quantum mechanically, reactions obey the inseparability principle [2] so that the product particles, such as fragments produced by photodissociation, can not be found in pure states, but should be described in terms of the density matrix $\rho_{S}$ [3], the key consequence of the path. Evidently, the more we know about the measurable correlation characteristics of $\rho_{S}$ (known as bipolar state moments [4]), the more definitely it can be inferred how a photon breaks a hitherto stable molecule.

\footnotetext{
*Corresponding author

Email address: alex@ak1197.spb.edu (A.P. Kouzov)
} 
The states of the nascent particles are most directly accessed by spectroscopic techniques whose proficiency becomes unexcelled when a reaction itself is initiated by light [5]. An advent of the laser-induced fluorescence (LIF) techniques that occured about 40 years ago led to a first breakthrough in the photodissociation studies. The frequency- and polarization-resolved LIF spectra allow to directly monitor the directional properties of the angular momentum $(\mathbf{j})$ of the product states [6] and provide detailed Doppler patterns [7] of the recoil velocity $\mathbf{v}$, its correlation with $\mathbf{j}$, and - according to Dixon's theoretical picture [4] - a number of the double and triple correlations of $\mathbf{v}$ and $\mathbf{j}[8,9]$ with the dissociating transition dipole moment $\mu_{f i}$ of the parent molecule. Such vector correlations are direct consequences of the photodissociation path $[5,10,11,12,13,14]$ and serve as a touchstone for intuitive bond rupture models as well as impetus to progress in the photoreaction scattering theory, the forefront topics in chemical physics. Especially intriguing is an effect of the photofragment handedness (helicity) characterized by a nonvanishing mean value of $\langle(\mathbf{v}, \mathbf{j})\rangle$. In a wider sense, the helicity can be also associated with other correlations between the products of the first powers of $\mathbf{v}$ and $\mathbf{j}$ and the orientational characteristics of $\mu_{f i}$. To our knowledge, direct evidences of the rotational handedness of molecular photofragments obtained with plane-polarized photolysis lasers are still missing although such LIF studies revealed that $\mathbf{v}$ and $\mathbf{j}$ in some cases are strongly aligned. To prove the helicity, i.e. the predominance of the clockwise rotation around the recoil direction over the counterclockwise one, or vise versa, additional experimental arguments should be found. More simply, such findings would imply that the rototranslational motion of a certain amount of the nascent particles resembles that of bullets from rifle's barrel.

The latest advances in the photolysis studies are due to tandems of optical spectroscopy and time-of-flight mass-spectrometry implemented in the Resonantly Enhanced Multi-Photon Ionization (REMPI) devices which allow to produce 2D- and 3D-images of photofragment recoil velocity distributions (see, e.g., the review [15]) on which the $\mathbf{v}$-j correlations are imprinted. REMPI spectroscopy, especially linked with kinetic energy analysis of the resulting photoelectrons, continues to make enormous contributions to understanding of the photolysis mechanisms. Using REMPI, the electronic angular momenta orientation [16] (of $\mathrm{Cl}$ atoms produced at the $\mathrm{ICl}$ laser photolysis) and the atomic spin polarization (of $\mathrm{H}^{2} \mathrm{~S}$ photofragments $[17,18]$ ) were detected for the first time. As was theoretically shown [12], the helicity can arise either because of the interference between the electronic pathways when 
two or more dissociating continua with different symmetries are excited, or because of the excitation of a perpendicular vibronic transition.

However, the potential of much simpler, purely optical means is far from being exhausted. New LIF schemes exploiting circularly polarized dissociation and counter-propagating pump beams were theoretically explored by Alexander $[19,20]$ and then introduced to practice. In so doing, not only the atomic polarization $[21,22]$ of the HCL photofragments was detected but the first data on the rotational helicity of the $\mathrm{OH}$ radicals produced at the $355 \mathrm{~nm}$ photolysis of hydrogen peroxide [19] were obtained as well.

Among other all-optical devices, Resonant Four-Wave Mixing (RFWM) spectroscopy should be named for its unique characteristics making it a novel, suitable tool to photolysis studies. To generate a RFWM signal beam at $\omega_{4}=\omega_{1}-\omega_{2}+\omega_{3}$, three input laser beams (1,2, and 3) [23] are focused into a small volume at a specially designed geometry. The signal beam is as coherent and well collimated as the input beams and can be, therefore, fully captured by a detector, in contrast to spontaneous emission collection in LIF. Its intensity $I_{4}$ is proportional to the squared modulus of the third-order susceptibility $\chi^{(3)}$ and is tremendously enhanced when the input frequencies are tuned to molecular dipole-allowed transitions. Owing to such enhancement, RFWM was successfully used $[24,25]$ to derive scalar characteristics of photolysis products at number densities as low as $10^{12} \mathrm{~cm}^{-3}$. These pioneering studies generated considerable interest and, though detection of the finer anisotropic effects remained then problematic, a review of the day [26] optimistically predicted that the RFWM technique "will prove very sensitive to angular-momentum polarization" and that it "will be applied to the probing of anisotropic angular momentum distributions, adding another valuable optical technique to the more commonly used LIF and REMPI methods." Indeed, RFWM has four controllable polarizations whereas LIF possesses only two and, even when operating with plane-polarized beams, all angularmomentum moments of order $L$ with $0 \leq L \leq 4$ are feasible for RFWM while only even-order moments $(L=0,2,4)$ can be tested by LIF at the same conditions. The separation of the laboratory-frame $L=1$ term can be principally achieved by LIF with elliptically polarized beams $[27,28]$ though at the expense of the setup complication. Anyway, the helicity derivation requires a next step - the Doppler-shape spectral analysis - which, to our knowledge, has not been done.

The $L=1$ moment is far directly accessed by RFWM whereas the relevant Doppler shapes are obtained by merely scanning the input frequency. 
Also, the RFWM responses are automatically state-resolved, both for the one- (or Degenerate FWM operating between a pair of molecular states) and Two-Color (TC, involving three states) variants while such resolution in the conventional LIF setup requires a spectrometer. In contrast to LIF, REMPI and DFWM, the use of the double-resonance TC-RFWM techniques immensely enhances the spectral selectivity allowing to resolve congested spectra [29] which is especially important for studies of molecular photofragments. Another RFWM distinction from LIF and REMPI is that the channels contributing to the on-resonance susceptibility $\chi_{\text {res }}^{(3)}$ are interfering, an intrinsic RFWM feature which can markedly show up in the integrated intensities and shapes alike.

Apart from the overall setup simplicity, RFWM as compared to REMPI gains also in the polarization device flexibility that allows to directly separate signals due to the orientational anisotropy of $\mathbf{j}$. However, the REMPI velocity images contain much richer information than the RFWM Doppler shapes which are determined by the mean value of $\mathbf{v}$ (see below). We notice also that RFWM is an efficient tool not only for molecular beams but for in situ measurements as well whereas REMPI applies solely to collisionless media. To summarize briefly, both techniques seem to complement each other to some extent.

So far, the DFWM [30, 31] and TC-RFWM [32, 33, 34] theory has been completed only for the rotationally isotropic states. The first theoretical and experimental DFWM studies of the anisotropy induced by optical pumping were undertaken by Vaccaro and coworkers [35, 36, 37, 38]. Very recently, DFWM [39] has been used for the first time to detect the anisotropy of the $\mathrm{OH}$ radicals at the $\mathrm{H}_{2} \mathrm{O}_{2}$ photodissociation by a plane-polarized radiation at $266 \mathrm{~nm}$ and to separate the $L=1$ components whose spectral shapes were indicative of the $\mathrm{OH}$ helicity [40]. The further progress is plagued by the lack of an exhaustive theory. To fill the gap, we provide in this Letter a theoretical groundwork for the RFWM probing of photofragments. In so doing, the conventional weak-field approximation $[30,31,32,33,34,41]$ is exploited.

The key issue is the derivation of $\chi_{\text {res }}^{(3)}$ which fully accounts for the rotational and translational anisotropy of nascent particles. To this end, the line-space (LS) Liouville formalism is the most appropriate tool which allowed a rigorous account for the collisional relaxation and proved thereby its superiority [33, 34] over the conventional approaches [30, 31, 32] to isotropic systems. Advantageuously, the LS derivations are done in a controllable, 
step-by-step manner which substantially facilitates cumbersome algebra in the anisotropic case. They are naturally combined with the irreducible spherical tensors (IST) machinery [42], a powerful tool to decouple different tensorial characteristics from $\chi_{\text {res }}^{(3)}$. As a result, the latter is converted into a sum of weighted products of the field, reaction path and molecular characteristics, i.e., into the form optimal for comparison with measured intensities.

Photofragment density matrix.-The key input characteristics of the photolytic $f \leftarrow i$ transition are the excess $\Delta \varepsilon$ of the photon energy above the dissociation threshold and $\mu_{f i}$ which, taken together, determine the reaction path. The detected RFWM intensity $I_{4}$ depends on the unit polarization vector $\mathbf{e}_{D}$ (hereafter assumed to be plane-polarized) of the photolysis laser and on the polarization states of the input and signal beams. Besides, the effect of the $\mu_{f i}-\mathbf{v}-\mathbf{j}$ correlations on the signal shapes is to be elucidated as well as the behavior of $I_{4}$ vs the set of $\left\{J_{k}\right\}$ of the angular momentum quantum numbers of the resonant states.

The LS consists of quantum operators $A, B, \ldots$ acting on the photofragment states with the metric $\langle\langle A \mid B\rangle\rangle=\operatorname{Tr} \rho_{0} A^{\dagger} B$ defined with the help of the isotropic part $\rho_{0}$ of $\rho_{S}$. The orthogonal and normalized LS basis consists of the symmetry-adapted $n \leftarrow m$ transition operators $K_{Q}^{(L)}(m, n)$ which are ISTs of rank $L[42]$

$$
K_{Q}^{(L)}(m, n)=\rho_{m m}^{-1 / 2} \sum_{M_{m} M_{n}}(-1)^{J_{m}-M_{m}} C_{J_{n} M_{n} J_{m}-M_{m}}^{L Q}\left|\alpha_{n} J_{n} M_{n}\right\rangle\left\langle\alpha_{m} J_{m} M_{m}\right|
$$

Here, $\left(\alpha_{k}, J_{k}\right)$ denotes a set of the principal quantum numbers corresponding to the state $k(=m, n), J_{k}$ and $M_{k}$ are the quantum numbers of the total angular momentum and its projection onto the $O Z$ axis in the laboratory frame (LF); $C_{b \beta c \gamma}^{a \alpha}$ is the Clebsch-Gordan coefficient, and $\rho_{m m}$ is the diagonal element of $\rho_{0}$. In what follows, we assume $\rho_{S}$ to be diagonal with respect to $\alpha$ and $J$, but not to $M$; therefore, $\rho_{S}$ can be expanded in $K_{Q}^{(L)}(m, m)$ (三 $\left.N_{Q}^{(L)}(m)\right)$. To incorporate the velocity anisotropy, the LS basis vectors should be multiplied by $C_{\sigma}^{(L)}\left(\Omega_{v}\right)$, the Racah harmonics depending on the orientation $\Omega_{v}$ of $\mathbf{v}$ in $\operatorname{LF}(X, Y, Z)$. Since $\rho_{S}$ is invariant under rotations of $\mathrm{LF}$, it can be written as a linear combination of the conventional scalar products [42] (denoted by $(. ., .)$.$) of two ISTs of the same rank (\lambda)$, i.e.,

$$
\rho_{S}=\sum_{i} \sum_{K L \lambda} \rho_{i i}^{3 / 2} \sqrt{2 J_{i}+1}\left(B_{K L}^{(\lambda)}(i, v),\left\{N^{(L)}(i) \otimes C^{(K)}\left(\Omega_{v}\right)\right\}^{(\lambda)}\right)
$$


where $\left\{A^{(a)} \otimes B^{(b)}\right\}^{(c)}$ denotes the contraction [42] of ISTs $A^{(a)}$ and $B^{(b)}$ into an IST of rank $c$, and $v=|\mathbf{v}|$. Since in the classical limit the matrix elements of $N_{Q}^{(L)}$ tend to the Racah harmonics $C_{Q}^{(L)}$ depending on the space orientation of $\mathbf{j}$, Eq. (2) is a quantum extension of the classical bipolar expansion [4]. Following Dixon [4], the LF bipolar IST $B_{K L}^{(\lambda)}(i, v)$ must be expressed via its (scaled) $z-$ component $b_{K L, 0}^{(\lambda)}(i, v) \equiv b_{\lambda K L}\left(O z \| \mu_{f i}\right)$ in the molecule-fixed frame, and after averaging over molecular orientations we arrive at the invariant expression

$$
\begin{aligned}
& \rho_{S}=\sum_{i L}\left(T^{(L)}, N^{(L)}(i)\right) \\
& T^{(L)}=\sum_{K \lambda=0,2} \rho_{i i}^{3 / 2} \sqrt{\left(2 J_{i}+1\right)} b_{\lambda L K}\left\{C^{(\lambda)}\left(\Omega_{D}\right) \otimes C^{(K)}\left(\Omega_{v}\right)\right\}^{(L)}
\end{aligned}
$$

in which the spherical angles $\Omega_{D}$ and $\Omega_{v}$ specify the orientations of $\mathbf{e}_{D}$ and $\mathbf{v}$ in LF, correspondingly. Since $\rho_{S}$ is not changed when the direction of $\mathbf{e}_{D}$ is reversed, $(-1)^{K+L}=1$ should hold.

As it follows from the ensuing discussion, $L$ coincides with the rank of the polarization IST $G_{a b c 4}^{(L)}$ built up from four unit polarization vectors $\left(\mathbf{e}_{k}\right)$ of the RFWM beams; hence, $L$ runs $0,1,2,3,4$. In (4), the momenta addition rules dictate that for the $\Omega_{D}$-independent $\lambda=0$ term, $K$ equals $L$ and varies in the same fashion. At $\lambda=2$ and fixed $L, K$ may vary from $|L-2|$ up to $|L+2|$ with the unity step. Hence, the maximal value of $K$ is 6 , the same as for LIF. However, when plane-polarized photons are used in the LIF excitation and detection channels, values of $L$ are restricted to $0,2,4$, and hence $K$ may vary from 0 up to 6 with step of 2 . In other words, combinations of odd $K$ and $L$ are forbidden in this case and the helicity remains silent at any dissociation beam polarization.

Third-order resonant susceptibility. - In the LS treatment of rotationally isotropic gases [33], $\chi^{(3)}$ is written as an LS matrix element of the product of three Liouville operators $\hat{V}_{k}(k=1,2,3)$ corresponding to dipole couplings with the input photons; this structure is preserved in the anisotropic case. The key difference lies in the calculation of the first matrix element $M_{1} \equiv\left\langle\left\langle\rho_{S} \rho_{0}^{-1}\right|\right.$ $\left.V_{a}\left|K_{1 \sigma_{1}}^{\left(l_{1}\right)}\right\rangle\right\rangle=\operatorname{Tr} \rho_{S} V_{a} K_{1 \sigma_{1}}^{\left(l_{1}\right)}$. However, at low gas pressures the resonances do not overlap which allows to apply the diagonal approximation for products of the LS matrix elements [33]. In particular, the product of $M_{1}$ and the second LS matrix element $\left\langle\left\langle K_{1 \sigma_{1}}^{\left(l_{1}\right)}\left|V_{b}\right| K_{2 \sigma_{2}}^{\left(r_{2}\right)}\right\rangle\right\rangle$ can be analytically summed over $\sigma_{1}$ and $l_{1}$. In doing so, the polarization unit vectors $e_{a}^{(1)}\left(=\mathbf{e}_{a}\right)$ and $e_{b}^{(1)}\left(=\mathbf{e}_{b}\right)$ 
should be coupled together to form an IST $\left\{e_{a}^{(1)} \otimes e_{b}^{(1)}\right\}^{\left(r_{1}\right)}\left(r_{1}=0,1,2\right)$ which enables summation over $l_{1}$. A general, off-resonant expression is a sum $\left(\sum\right)$ over permutations of the input photons $a, b, c$ (which corresponds to different time orderings of the photon-matter interactions [41]), all virtual transitions, and over the tensor ranks $r_{1}, r_{2}$, and $L$ :

$$
\begin{aligned}
& \chi^{(3)}=C \sum d_{m_{1} n_{1}} d_{n_{3} m_{3}}(-1)^{J_{m_{2}}+J_{n_{2}}+J_{n_{1}}+J_{m_{1}}+r_{2}}\left(T^{(L)}, G_{a b c 4}^{(L)}\left(r_{1}, r_{2}\right)\right) R_{K_{1}}\left(\omega_{a}^{\prime}\right) R_{K_{2}}\left(\omega_{a}^{\prime}+\omega_{b}^{\prime}\right) \\
& \times R_{K_{3}}\left(\omega_{4}^{\prime}\right)\left\{(-1)^{r_{2}} \delta_{m_{1} m_{2}} d_{n_{1} n_{2}}\left[\rho_{m_{1} m_{1}} S_{1}-\rho_{n_{1} n_{1}} S_{3}\right]-\delta_{n_{1} n_{2}} d_{m_{2} m_{1}}\left[\rho_{n_{1} n_{1}} S_{2}-\rho_{m_{1} m_{1}} S_{4}\right]\right\} \\
& \times\left[\delta_{m_{2} m_{3}}(-1)^{r_{2}} T_{n_{2} n_{3} m_{2}}^{r_{2}} d_{n_{2} n_{3}}-\delta_{n_{2} n_{3}} T_{n_{2} m_{3} m_{2}}^{r_{2}} d_{m_{3} m_{2}}\right]
\end{aligned}
$$

where $d_{m n}$ are the reduced matrix elements of the photofragment dipole moment $\mathbf{d}, G_{a b c 4}^{(L)}\left(r_{1}, r_{2}\right)=\left\{\left\{e_{a}^{(1)} \otimes e_{b}^{(1)}\right\}^{\left(r_{1}\right)} \otimes\left\{e_{c}^{(1)} \otimes e_{4}^{*(1)}\right\}^{\left(r_{2}\right)}\right\}^{(L)}$, and $T_{i j k}^{r}$ are the $6 \mathrm{j}$-symbols defined in Ref. [34]. We shall not specify the constant factor $C$ since only relative intensities are of prime interest. Notice that one of the polarization vectors $e_{j}^{(1)}(j=a, b, c)$ refers to beam 2 replenished by generated photons and should be complex conjugated (as shown in $G_{a b c 4}^{(L)}$ for the output photon 4) whereas the corresponding Doppler-shifted frequency $\omega_{j}^{\prime}=\omega_{j}-\left(\mathbf{k}_{j}, \mathbf{v}\right)$ (with the wave vector $\mathbf{k}_{j}$ ) should be negated. The resonance factors $R_{K_{l}}\left(\omega_{j}^{\prime}\right)=\left[\omega_{j}^{\prime}-\omega_{K_{l}}+i \gamma_{K_{l}}\right]^{-1}$ contain the frequency $\omega_{K_{l}}$ and the damping constant $\gamma_{K_{l}}$ of the $K_{l}$ (i.e., $\left.n_{l} \leftarrow m_{l}\right)$ transition. The rank $r_{2}\left(r_{2}=\right.$ $0,1,2)$ appears when the polarization vectors $e_{c}^{(1)}$ and $e_{4}^{(1)}$ are decoupled from $\hat{V}_{c}\left(\mathbf{d}, \mathbf{e}_{4}^{*}\right)$.

The derived coefficients $S_{1}$ and $S_{4}$ in (5) are expressed via $6 \mathrm{j}$-symbols:

$S_{1}=(-1)^{L+r_{1}+r_{2}-J_{n_{1}}+J_{n_{2}}}\left\{\begin{array}{ccc}J_{m_{1}} & r_{1} & J_{n_{2}} \\ 1 & J_{n_{1}} & 1\end{array}\right\}\left\{\begin{array}{ccc}J_{m_{1}} & r_{1} & J_{n_{2}} \\ r_{2} & J_{m_{1}} & L\end{array}\right\}$ while $S_{2}$ and $S_{3}$ are equal to 9 j-symbols: $S_{2}=\left\{\begin{array}{ccc}J_{n_{1}} & r_{2} & J_{m_{2}} \\ 1 & r_{1} & 1 \\ J_{m_{1}} & L & J_{m_{1}}\end{array}\right\} ; S_{4}$ and $S_{3}$ are obtained from $S_{1}$ and $S_{2}$, respectively, by interchanging the state labels $n_{k}$ and $m_{k}(k=1,2)$. The different functional dependences of $S_{n}$ (cf. $S_{1}, S_{4}$ and $\left.S_{2}, S_{3}\right)$ on $\left\{J_{k}\right\}$ are explained by molecular gratings formed in the ground $(i)$ and excited $(f)$ electronic states after an interaction with a pair of the pump photons of the same frequency. As this takes place, the initial LS vector $|i\rangle\langle i|$ can be converted either into $|f\rangle\langle f|$ - and then the upperstate grating is said to be formed - or remain unchanged (the case of the ground-state grating). Since only the $i$-state is anisotropic, the gratings are different and thus differently affect $\chi^{(3)}$. The effect disappears for the 
isotropic $L=0$ term for which $S_{k}$-functions reduce to a single $6 \mathrm{j}$-symbol and the previous results $[32,34,33]$ are reproduced. Structurally, the $\chi^{(3)}$ expressions obtained in the isotropic [33] and anisotropic cases are similar and, therefore, the resonance terms can be separated from (5) in the same fashion as used before [33].

Generally, several polarization ISTs $G_{a b c 4}^{(L)}$ corresponding to different time orderings enter $\chi^{(3)}$, which plagues an extraction of the correlation parameters from experimental data. However, in the collision-free limit it is possible to recouple ISTs in such a way that at the given $L$ only one IST, viz., $G_{1234}^{(L)}$, enters the final expression. The proof will be given in an extended publication.

Velocity averaging. - In contrast to the frequency-resolved LIF, where the frequency detuning from the line center equals the Doppler shift, the integration of $\chi_{\text {res }}^{(3)}$ over the recoil directions $\mathbf{v}$ is not trivial. This will be done for the DFWM case (all input frequencies are the same $\left(\omega_{j}=\omega ; j=1,2,3,4\right)$ and operate near the $n \leftarrow m$ transiton) assuming that the photodissociation energy excess $\Delta \varepsilon$ is completely converted into the recoil energy $m v_{0}^{2} / 2$, i.e., $b_{\lambda K L}(v ; i)=f_{\lambda K L}\left(v_{0} ; i\right) \delta\left(v-v_{0}\right)$. The task is then reduced to the 1Dintegration of the product of $C_{0}^{(L)}\left(\Omega_{v}\right)$ and three resonance factors $R_{K_{l}}$ over $v_{Z}$, the velocity projection onto $O Z$. For the used DFWM geometry $[39,40]$, all four beams were nearly copropagating along the common axis $\mathbf{k} \| O Z$ so that the Doppler shift $\left(\left(\mathbf{k}_{a}-\mathbf{k}_{b}\right), \mathbf{v}\right)$ in $R_{K_{2}}$ disappears. The integral can be then expressed via the Legendre functions of complex variable. However, in the high-recoil limit $\gamma_{n m} / k v_{0} \rightarrow 0$ one can close the integration path by the semicircle of the radius $v_{0}$ in the complex upper half plane and take the integral by residues with a negligible loss of accuracy. While the pole $z_{3}$ of $R_{K_{3}}$ always lies in the upper half plane, the position $z_{a}$ of the $R_{K_{1}}$ singularity depends whether the first photon (photon $a$ ) is emitted (case A, for which $\omega_{a}<0$ and hence $\left.I m z_{a}<0\right)$ or absorbed $\left(\omega_{a}>0, I m z_{a}>0\right.$, case B). In case $B$, the residue contributions cancel each other and the integral vanishes. The latter does not vanish only in case A and we obtain the expression

$$
\begin{aligned}
\chi_{\text {res }}^{(3)}= & \sum_{\lambda=0,2} \sum_{K L r_{1} r_{2}} F\left(r_{1}, r_{2}, L ;\left\{J_{k}\right\}\right) f_{K L N}\left(v_{0}, i\right) \\
& \times\left\{C^{(\lambda)}\left(\Omega_{D}\right) \otimes G_{a b c 4}^{(L)}\left(r_{1}, r_{2}\right)\right\}_{0}^{(K)} P_{K}\left(\Delta \omega / k v_{0}\right)
\end{aligned}
$$

in which the allowed photon orderings are 2134 and 2314. The explicit form of the $F$-function can be straightforwardly derived from Eq. (5). Besides, 
the argument $\left(\omega-\omega_{n m}\right) / k v_{0}$ of the Legendre polynomial $P_{K}$ should not exceed unity - otherwise $z_{3}$ crosses the integration semicircle and the integral disappears.

The high-recoil damping effect becomes evident in the time domain where a term oscillating with the frequency $2 k v_{z}$ appears in case $\mathrm{B}$ and is washed out by the velocity averaging; in contrast, such oscillations are absent in case A. Remarkably, similar damping occurs also in an isotropic gas when the Doppler effect becomes prevailing over the collisional broadening [33].

Polarization tensors. - To derive all $2 L+1$ components of the IST $G_{a b c d, \sigma}^{(L)}$, cumbersome triple contractions of the polarization unit vectors are to be done. Fortunately, the collinear beam geometry drastically alleviates the calculation. From Eq. (6) and properties of the Racah harmonics [42], it follows that only the $\sigma=0, \pm 2$ components can contribute to $\chi_{\text {res }}^{(3)}$. Some results for plane-polarized beams are outlined below; a comprehensive analysis will be published later. The properties of the scalar term $G_{a b c d}^{(0)}$ are well known from the previous studies [33] and will not be considered here.

At $L=1$, the $r_{1}=r_{2}=1$ term of $G_{a b c d}^{(1)}$ vanishes whereas other $\left(r_{1}, r_{2}\right)$ terms, viz $(0,1),(1,0),(1,2),(2,1)$, and $(2,2)$ ones, contribute to the sole nonzero and imaginary component, $G_{a b c d, 0}^{(1)}$. The real $G_{a b c d, 0}^{(2)}$ component is contributed by the $(1,1),(0,2),(2,0),(2,2)$ terms as well as by the $(1,2)$ and $(2,1)$ ones. The complex-valued components $G_{a b c d, \pm 2}^{(2)}$ are built up by $(0,2)$, $(2,0),(1,2),(2,1)$, and $(2,2)$ terms. The $(1,2),(2,1)$ and $(2,2)$ terms determine the $L=3$ IST which has the imaginary component $G_{a b c d, 0}^{(3)}$ and the complex ones $G_{a b c d, \pm 2}^{(3)}$. Finally, at $L=4$ only the $(2,2)$ term is allowed and generates the real $G_{a b c d, 0}^{(4)}(2,2)$ and complex-valued $G_{a b c d, \pm 2}^{(4)}(2,2)$ components.

Polarization dependence of $\chi_{\text {res }}^{(3)}$ - Using $(6), \chi_{\text {res }}^{(3)}$ can be calculated at any polarization geometry. The most striking manifestation of the rotational anisotropy is the signal appearance at polarizations inactive in the rotationally isotropic cases. This was indeed observed in the DFWM experiments $[39,40]$ with plane-polarized beams at the "forbidden" polarization configuration $\left(\mathbf{e}_{1}, \mathbf{e}_{2}, \mathbf{e}_{3}, \mathbf{e}_{4}\right)=(Y Y Y X)$ with $X O Y$ plane perpendicular to $O Z \| \mathbf{k}$. Since $\left(\mathbf{k}, \mathbf{e}_{D}\right)=0$, the nonvanishing components of $C_{\sigma}^{(\lambda)}\left(\Omega_{D}\right)$ with $\sigma=0, \pm 2$ produce terms varying as $e^{i \sigma \varphi}$ where $\varphi$ is the angle between $\mathbf{e}_{D}$ and $O X$. The intensity vs $\varphi$ behaviour can be rationalized from Eq. (6). Due to the geometrical simplicity, only few, purely imaginary $G$-tensor elements can contribute to $\chi^{(3)}(Y Y Y X)$, viz., $G_{0}^{(1)}, G_{-2}^{(2)}=-G_{2}^{(2)}, G_{0}^{(3)}, G_{-2}^{(3)}=G_{2}^{(3)}$, and 


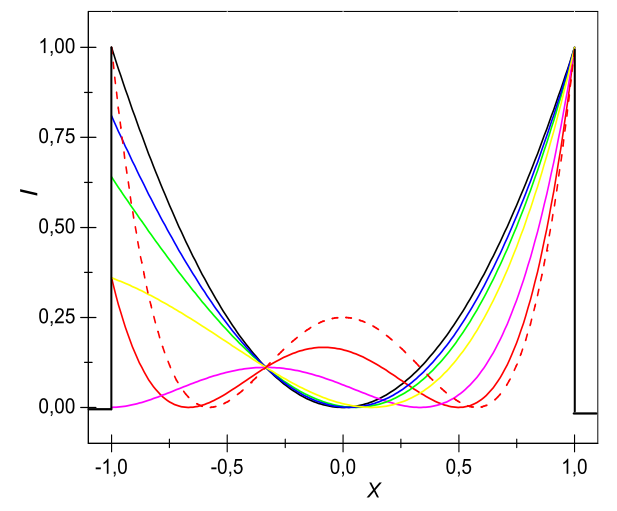

Figure 1: RFWM Doppler shapes $I(x)\left(x=\left(\omega-\omega_{0}\right) / k v_{0} ;|x| \leq 1\right)$ calculated at different weights $w_{1}$ and $w_{2}=1-w_{1}$ of the first- and second-degree Legendre polynomials $P_{K}(x)$ : $w_{1}=1$ (black line), $w_{1}=0.95$ (blue), $w_{1}=0.90$ (green), $w_{1}=0.80$ (yellow), $w_{1}=0.5$ (magenta), $w_{1}=0.20$ (solid red line), $w_{1}=0$ (dashed red line).

$G_{-2}^{(4)}=-G_{2}^{(4)}$. The final expression reads

$$
\chi_{\text {res }}^{(3)} \propto A_{0}+\cos (2 \varphi) A_{2}+i \sin (2 \varphi) B_{2}
$$

where $A_{\lambda}(\lambda=0,2)$ are linear combinations of odd-degree polynomials $P_{K}$ $(K \geq 1)$ weighted by products of $f_{\lambda L K}$ with odd $L$ and certain real coefficients depending on $\left\{J_{k}\right\} ; B_{2}$ is built up in the same fashion, but with even-degree $P_{K}(K \geq 2)$ and $f_{2 L K}$ with even $L \geq 2$.

Therefore, the signal at $\varphi=0$ is entirely produced by the helicity, i.e., by correlations between odd powers of $\mathbf{j}$ and $\mathbf{v}$. Since $I_{4} \sim\left|\chi_{\text {res }}^{(3)}\right|^{2}$, the helicityinduced Doppler profiles at $\varphi=0$ are symmetric doublets with the disappearing intensity in the center. Evidently, when the $B_{2}$-terms are vanishing, such shape form is not affected by $\varphi$ and, besides, $I_{4}(-\varphi)=I_{4}(\varphi)$.

A fundamental theoretical conclusion $[12,13]$ is that the rotational orientation can arise only when two (or more) continuum states with different helicities are simultaneously excited. The photofragment helicity is thus a fingerprint of the interference between the dissociation channels. In general, the bipolar moments as well as $A_{\lambda}(\lambda=0,2)$ and $B_{2}$ may be complex. In this case, asymmetric Doppler shapes at finite $\varphi$ appear and, besides, $I_{4}(-\varphi) \neq I_{4}(\varphi)$. Generally, the $I_{4}$ vs $\varphi$ behavior gives a direct information 
on relative contributions from $A_{0}, A_{2}$ and $B_{2}$. When the $A$-terms are negligible, $I_{4}(\varphi)=I_{4}(-\varphi)$ holds, the shapes are again symmetric, but the central dips are smoothed.

Thus, the asymmetry of the Doppler shapes arises when several Legendre polynomials of odd and even degrees jointly contribute to the real and imaginary parts of $\chi_{\text {res }}^{(3)}$ and thereby interfere in $\left|\chi_{\text {res }}^{(3)}\right|^{2}$. As seen from Eq. 7, such interference becomes allowed only when the $A_{\lambda}$ and $B_{2}$ coefficients are complex. On the one hand, the profile asymmetry indicates the rotational helicity per se and, on the other hand, can be used to establish the sign of $\langle(\mathbf{v}, \mathbf{j})\rangle$.

The simplest interference patterns are given on Fig. 1 depicting transformation of the RFWM Doppler shapes with varying weights $w_{1}$ and $w_{2}=$ $1-w_{1}$ of the first- and second-degree Legendre polynomials $P_{K}(K=1,2)$. When the weights vary in the $(0,1)$ interval, the shapes are unity normalized at the largest dimensionless detuning $x=\left(\omega-\omega_{0}\right) / k v_{0}=1$ and have an enhanced "blue" shoulder provided $w_{1}$ is distinct from 0 or 1 . When the helicity effects are predominant $\left(w_{1} \rightarrow 1\right)$, a parabolic shape $I=x^{2}$ is formed so that the intensity disappears at $x=0$. In contrast, in the pure alignment case (i.e. at $w_{1}=0$ ) a central maximum with the intensity $I(0)=0.25$ emerges whereas the satellites at $x= \pm 1$ become sharper. As seen from Fig. 1, a noticeable asymmetry of the helicity-induced shapes arises at fractions $w_{2}$ of the alignment terms as small as $\approx 0.05$. Positiveness of the coefficients $w_{1}$ and $w_{2}$ warrants the enhancement of the high-frequency shoulder relative to its "red" counterpart. By contrast, the high-frequency part is dampened if we take $w_{1}$ from the $(-1,0)$ interval and set $w_{2}=1+w_{1}$. In this case, profiles which are mirrow symmetric to those on Fig. 1 appear.

Another peculiarity also owing to the coherent nature of RFWM may manifest when coincidently generated photofragments are the same as it occurs, for instance, in photodissociation of $\mathrm{H}_{2} \mathrm{O}_{2}$. Provided such fragments are in the same quantum states, the total $\chi_{\text {res }}^{(3)}$ becomes a sum of the fragment susceptibilities and interference between them can reveal itself in the RFWM signals. In so doing, the LIF results [43] testifying to (scalar) correlations between the fragment populations can be extended to nonscalar cases thus leading to a finer state of the art.

Acknowledgments. - Support from the Swiss National Science Foundation (grant 200021_153170/1) and from the Russian Foundation for Basic Research (grant 15-03-04997) is gratefully acknowledged. 
[1] P. Atkins and J. de Paola, Physical Chemistry (Oxford University Press, Oxford, 2006)

[2] B. de Espagnat, Conceptual Foundations of Quantum Mechanics (Perseus Book Publishing, Reading, 1999).

[3] K. Blum, Density Matrix: Theory and Applications (Springer Verlag, Berlin, 2012).

[4] A.N. Dixon, J. Chem. Phys. 85 (1986) 1866-1879.

[5] H. Sato, Chem. Rev. 101 (2001) 2687-2725.

[6] C.H. Greene, R.N. Zare, J. Chem. Phys. 78 (1983) 6741-6753.

[7] M. Dubs, U. Brühlman, J.R. Huber, J. Chem. Phys. 84 (1986) 31063119.

[8] K.-H. Gericke, S. Klee, F. Comes, R.N. Dixon, J. Chem. Phys. 85 (1986) 4463-4479.

[9] M.P. Docker, A. Hodgson, J.P. Simons, Chem. Phys. Lett. 128 (1986) 264-269.

[10] P.L. Houston, J. Phys. Chem. 91 (1987) 5388-5397.

[11] A.J. Alexander, R.N. Zare, Acc. Chem. Res. 33 (2000) 199-205.

[12] L.D.A. Siebbeles, M. Glass-Maujean, O.S. Vasyutinskii , J.A, Beswick, O. Roncero, J. Chem. Phys. 100 (1994) 3610-3623.

[13] T.P. Rakitzis, R.N. Zare, J. Chem. Phys. 110 (1999) 3341-3350.

[14] M.L. Costen, S. Marinakis, and K.G. McKendrick, Chem. Soc. Rev. 37, 732 (2008).

[15] A. G. Suits, O. S. Vasyutinskii, Chem. Rev. 108 (2008) 3706-3746.

[16] T.P. Rakitzis, S.A. Kandel, A.J. Alexander, Z.H. Kim, R.N. Zare, Science 281 (1998) 1346-1349.

[17] T. P. Rakitzis, P. C. Samartzis, R. L. Toomes, T. N. Kitsopoulos, A. Brown, G. G. Balint-Kurti, O. S. Vasyutinskii, J. A. Beswick, Science 300 (2003) 1936-1938. 
[18] B. M. Broderick, V. Y. Chernyak, A. G. Smolin, O. S. Vasyutinskii, A. G. Suits, Chem. Phys. Lett. 635 (2015) 350-354.

[19] A.J. Alexander, J. Chem. Phys. 118 (2003) 6234-6243.

[20] A.J. Alexander, J. Chem. Phys. 123 (2005) 194312:1-9.

[21] D. Sofikitis, L. Rubio-Lago, L. Bougas, A. J. Alexander, T. P. Rakitzis, J. Chem. Phys. 129 (2008) 144302:1-10.

[22] D. Sofikitis, L. Rubio-Lago, A. J. Alexander, T. P. Rakitzis, Europhys. Lett. 81 (2008) 68002:1-4.

[23] It is implied that photons are absorbed in beams 1 and 3 and generated in beams 2 and 4.

[24] T.J. Butenhoff, E.A. Rohlfing, J. Chem. Phys. 98 (1993) 5460-5468; ibid. 98 (1993) 5469-5479.

[25] T.A.W. Wasserman, A.A. Arias, T. Müller, P.H. Vaccaro, Chem. Phys. Lett. 262 (1996) 329-336.

[26] A.J. Orr-Ewing, R.N. Zare, Annu. Rev. Phys. Chem. 45 (1994) 315-366.

[27] A.C. Kummel, G.O. Sitz, R.N. Zare, J. Chem. Phys. 88 (1988) 73577368 .

[28] Y. Mo and T. Suzuki, J. Chem. Phys. 109 (1998) 4691-4692.

[29] P.P. Radi, H.-M. Frey, B. Mischler, A.P. Tzannis, P. Beaud, T. Gerber, Chem. Phys. Lett. 265 (1997) 271-276.

[30] S. Williams, R.N. Zare, L.A. Rahn, J. Chem. Phys. 101 (1994) 10721092.

[31] S. Williams, L.A. Rahn, R.N. Zare, J. Chem. Phys. 104 (1996) 39473955.

[32] S. Williams, E.A. Rohlfing, L.A. Rahn, R.N. Zare, J. Chem. Phys. 106 (1997) 3090-3102.

[33] A. Kouzov and P. Radi, J. Chem. Phys. 140 (2014) 194302:1-13. 
[34] A.P. Kouzov, P.P. Radi, Phys. Rev. A 63 (2000) 010701:1-4.

[35] T.A.W. Wasserman, P.H. Vaccaro, B.R. Johnson, J. Chem. Phys. 108 (1998) 4-7.

[36] T. Müller, T.A.W. Wasserman, P.H. Vaccaro, B.R. Johnson, J. Chem. Phys. 108 (1998) 4-7.

[37] T.A.W. Wasserman, P.H. Vaccaro, B.R. Johnson, J. Chem. Phys. 108 (1998) 7713-7738.

[38] D. Murdock, L.A. Burns, P.H. Vaccaro, J. Phys. Chem. A 113 (2009) 13184-13198.

[39] P. Maksyutenko, P.P. Radi, D.N. Kozlov, A.P. Kouzov, J. Raman Spectrosc. 44 (2013) 1349-1355.

[40] A. Kouzov, P. Radi, P. Maksyutenko, D. Kozlov, Journal of Physics: Conference Series 548 (2014) 012016:1-5.

[41] S. Mukamel, The Principles of Nonlinear Optical Spectroscopy (Oxford University Press, Oxford, 1995)

[42] D.A. Varshalovich, A.N. Moskalev, V.K. Khersonskii, Quantum Theory of Angular Momentum (World Scientific, Singapore, 1988).

[43] K.-H. Gericke, H.G. Glaser, C.Maul, F.J. Comes, J. Chem. Phys. 92 (1990) 411-419. 


\section{Resonant Four-Wave Mixing Spectroscopy of Photofragments}

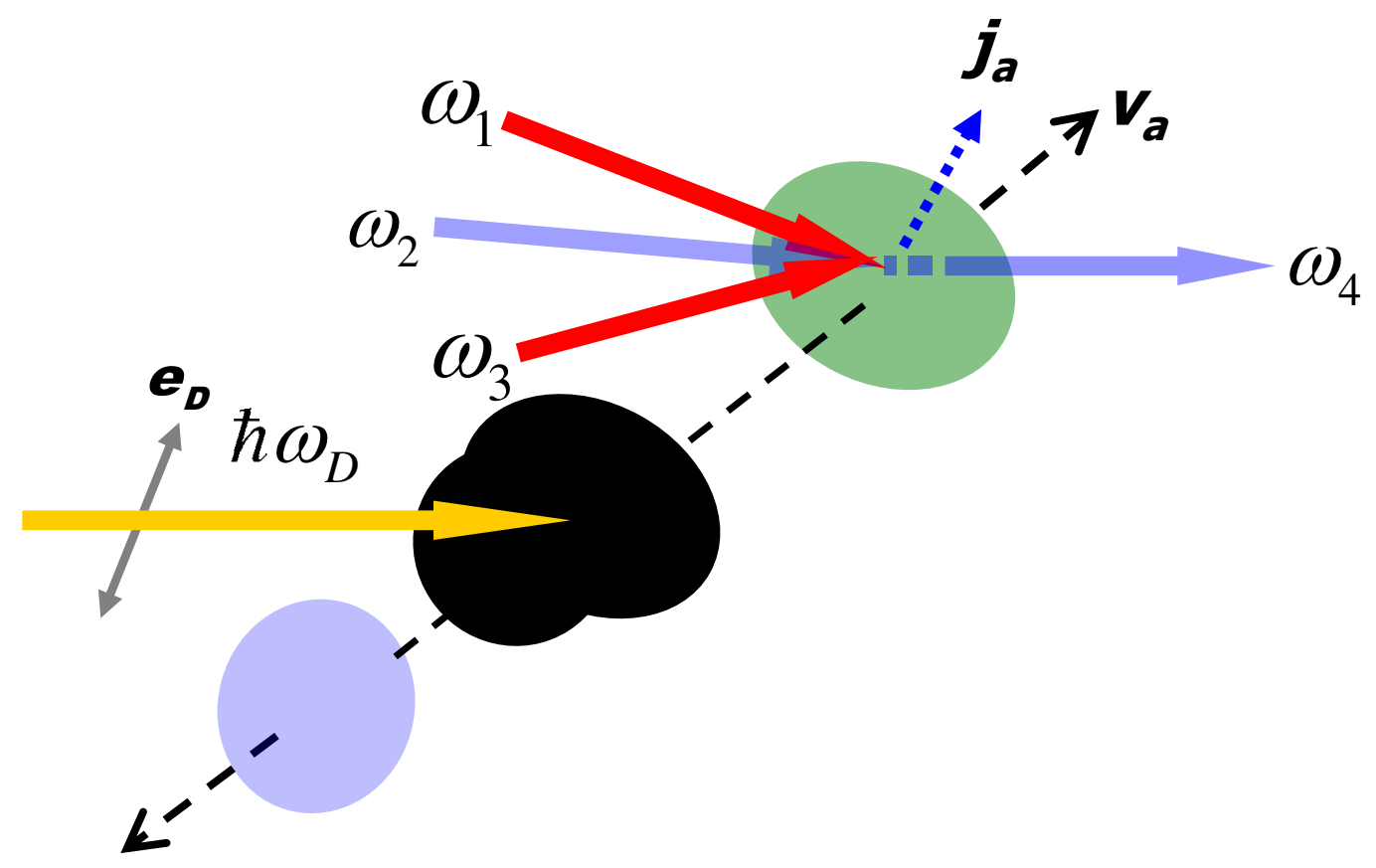

